\title{
Adaptive Control of Out-of-Sequence Packet Arrival for Optimization of Bandwidth Aggregation of Heterogeneous Communication Links
}

\author{
Syed Zubair Ahmad \\ Management Information System, Pakistan Institute of Nuclear Science and Technology, Islamabad, Pakistan
}

\section{Email address:}

zubair@pinstech.org.pk

\section{To cite this article:}

Syed Zubair Ahmad. Adaptive Control of Out-of-Sequence Packet Arrival for Optimization of Bandwidth Aggregation of Heterogeneous Communication Links. Advances in Networks. Vol. 3, No. 1, 2015, pp. 1-7. doi: 10.11648/j.net.20150301.11

\begin{abstract}
Multipath transmission of flows over heterogeneous communication channels faces numerous challenges in optimally utilizing combined communication resources for ubiquitous applications. One major issue is the out-of-sequence arrival of packets at receiver that commonly amplifies while distributing packets of a flow over multiple paths. These packets experience asymmetric path characteristics that results in diverse congestion states for ubiquitous traffic flows. This paper presents an adaptive mechanism for estimation of path suitability for a specific type of flow on the basis of end-to-end path delay statistics, packet drop and available bandwidth. The estimation process is used to develop path ranks for scheduling delay traffic on multiple paths with reduced probability of out-of-sequence arrival at the receiver. The proactive approach used in this scheme is computationally less expensive as compared to complex reordering of packets at the receiver. The results have shown robust performance of proposed mechanism in achieving acceptable quality-of-service levels during multipath transmission of flows with minimized out-of-sequence reception and reduced buffer occupancy.
\end{abstract}

Keywords: Out-of-Sequence Arrival, E2E Path Ranking, Heterogeneous Wireless Networks, Asymmetric Path Characteristics, Bandwidth Aggregation, Throughput Maximization

\section{Introduction}

The multipath transport of data streams over multiple available communication links of multimode devices has attracted keen interest of researchers working in the area of ubiquitous computing and mobile networks. It enables key benefits of increased bandwidth and induces quality attributes of redundancy, load balancing, data security and network congestion management at the terminal points [1]. The multipath transport; however faces major challenges in optimally unifying heterogeneous communication channels due to varying end-to-end (E2E) characteristics of each path [3]. The E2E delay variations cause out-of-sequence (OOS) arrival of packets at the receiver that generally induce complex packet reordering mechanisms before presentation to applications. The congestion control and packet retransmission strategies of connection oriented transport protocols; like transmission control protocol (TCP) and stream control transmission protocol (SCTP) face significant overhead due to repeated re-transmissions; resulting in much reduced throughput. Hence, stripping a traffic flow over multiple links requires either a pro-active or reactive strategy to minimize impact of E2E path asymmetries.

The E2E path characteristics are primarily influenced by path congestion and packet drop rates on the routers. These characteristics significantly vary during life cycle of a session. In pursuance of this problem, proactive strategies are generally considered less expensive as compared to their reactive counterparts [5]. The proactive strategies require adaptive estimation of E2E path characteristics before striping the flows on multiple links at the sender side. The task of estimation of these parameters though is not trivial as it requires complex modeling and numerical processing before achieving desirable convergence; it's processing load vestiges only on sender. Further, the complexity of problem may be reduced by making some simplifications at the cost of marginally reduced accuracy [4]. The trade-off in estimation process ensures timely convergence without lose of significant accuracy of estimated values. In contrast; a reactive strategy requires complex packet reordering 
mechanisms at the receivers and may still not be effective for real-time applications that are highly delay sensitive [2]. Experiments have shown that real-time application face high drop rate due to OOS arrival phenomenon [7].

In this paper, we have presented a proactive technique deployed at the sender and tailored to minimize OOS arrivals at receiver. It uses a simplified stochastic model to estimate E2E delay values using bounded variance network calculus (BVNC) [4]. Studies have found that most of the E2E characteristics can be mapped on E2E delay [10]. Hence, it may be chosen as a single parameter for describing E2E path ranking for simplifying problem at hand. In our approach, the estimated value of E2E delay; along-with packet drop rate and link throughput is used to choose the contribution of each available path for a specific type of data flow. The packets of that flow are then scheduled over best paths, keeping in view the delay and packet drop constraints of that flow. The procedure is applied for each flow requiring service. Ranking of E2E path is obtained through stochastic analysis of probe data. It is an adaptive mechanism that determines state of congestion on each network. Consequently; weights are assigned to the available paths for each class of competing flows. These weights keep on changing during the life cycle of flow. The aggregated bandwidth achieved through this method is highly modular and provides right granularity of bandwidth for competing flows. The cumulative result of traffic load distribution over multiple paths also improves aggregation ratio that makes key finding of this work. The proactive approach of multi-path transport of a flow is more suitable for the multipath transport protocols like SCTP and real-time transport protocol (RTP) as session-based deployment of this strategy can assist internal congestion and flow control processes of these protocols. In [1], we proposed stochastic model for estimation of E2E delay using 2-state Poisson process as shown in Figure 1. The rate of change of packet arrival and otherwise is represented by $\mu_{f} \& \lambda_{f}$ respectively; whereas $r_{f}$ is the rate requirement of flow. Each path comprises of different number of routers as shown in Figure 1.

The rest of the paper is organized as follows. Section II presents brief review of the various researches that suggest proactive strategies for multipath transport and bandwidth aggregation. Section III presents proposed adaptive OOS controlled multipath transport and path ranking technique. Section IV discussed analytical and simulation results of the proposed scheme. We conclude in Section V.

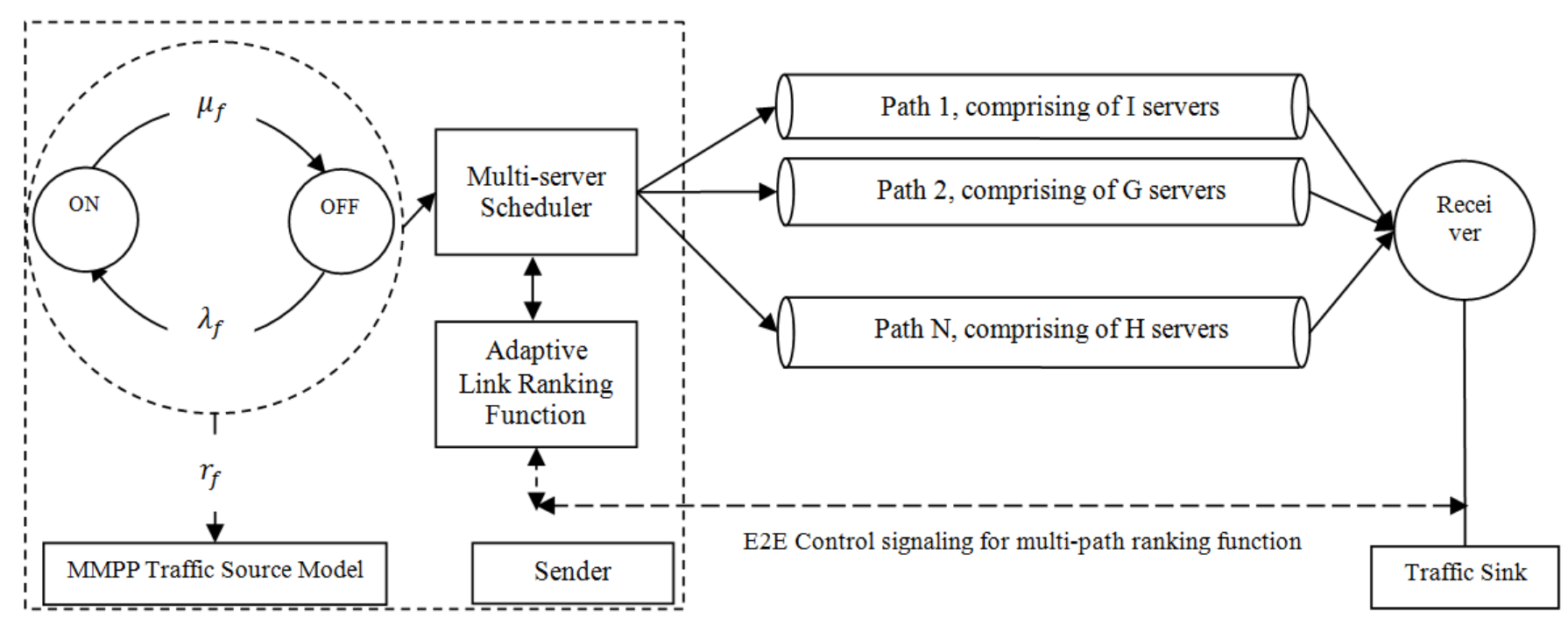

Figure 1. A system model for multi-path transport of MMPP traffic source [1].

\section{Related Work}

The topic of bandwidth aggregation (BAG) has some associated problems during multipath transport of data flows that may significantly reduce its potential benefits. The heterogeneous characteristics of each path induce variation in E2E delay that causes out-of-sequence (OOS) arrival of packets at the receiver. The problem of OOS arrival of packets has been largely studied in light of congestion control of connection oriented transport protocols like TCP and SCTP; but has much more profound impact over multipath transport of sessions using connection less protocols [8]. In this section, we discuss recent findings on the subject.

Serral-Gracia et al have studied the issue in a test-bed environment using European Academic Network (EAN). In this study, they have identified some performance degradation factors that need more analysis to suggest remedial actions [6]. These factors include random losses in connectionless protocols like user datagram protocol (UDP) and great amount of OOS arrival of packets. The impact of these factors has been characterized as catastrophic. In another research, Kristian Evensen et al, have proposed a proactive mechanism of monitoring throughput and delay statistics of heterogeneous paths to adjust the flow rate of a stream and distribution rate of flow on available paths [7]. They have used a buffer to hold packets at the proxy to match delay statistics of all paths. This reduces packet reordering and buffer requirements at the client. This approach though useful for client server computing models but may not be 
effective in peer-to-peer models. It also adds an additional proxy server to control multipath transport.

Jinghua Yan et al, have proposed an improved dynamic time warping (IDTW) method that aligns two feature vectors using non-linear alignment to improve the performance of link classification and subsequent multipath packet transport [11]. The accuracy of machine learning schemes for classification of link is important because of the subsequent packet transport decision making but the convergence time is more important in case of change in link characteristics. The time between the change in link characteristics and the convergence of machine learning scheme should be reasonably small to be useful. The larger convergence time may lead to serious degradation in minimizing OOS packets at the receiver.

$\mathrm{Wu}$, Jiyan et al, have studied the multipath transport and OOS packet arrival issue using load distribution model [15]. In this work, a sub-packet based multipath load distribution (SPMLD) has been proposed that splits network traffic at the granularity of sub-packet level. Although sub-packet level loads distribution minimizes transmission delays but adds packet reassembly and reordering overhead.

Sumet Prabhavat et al, have studied impact of multipath transport over TCP by proposing a modified random early detection (RED) for traffic load distribution over multiple paths [8]. TCP is highly sensitive to OOS arrival and its throughput greatly reduces in case of higher OOS rate. The proposed model takes advantage of RED queue management scheme to minimize buffer occupancy. The size of queues is also minimized for better flow management. Despite it novelty, the approached is not studied in light of congestion control.

Numerous other bandwidth aggregation techniques have shown that the cumulative impact of OOS packet arrival can be catastrophic for end-to-end (E2E) performance metric during life cycle of a session[9][10][12]\&[13]. The subsequent results of OOS arrival leads to higher retransmission and packet drop rates that may cause repeated triggering of congestion control mechanisms [2] \& [14]. Even in best case scenarios, the performance of BAG techniques may not coincide with the theoretical sum of all available capacities due to its operational and maintenance overheads. It is therefore, important to quantitatively analyze the concerned parameters that can make BAG techniques more useful and workable. The accuracy of such analysis can greatly facilitate process of developing suitable scheduling strategies and distribution of traffic load on available channels. The purpose of these scheduling strategies may be focused on subsiding counter-productive impact of heterogeneity of multiple paths and to achieve one or more desirable performance metrics; either for the individual flows or for the networks [13].

\section{Adaptive Control of OOS Arrival}

In our previous work [1] \& [3], both deterministic and stochastic models were presented to analyze path asymmetries during multipath transport of a data traffic flow. These models provide mathematical foundation for determining path characteristics and developing adaptive scheduling strategies. The scheduling task is than constrained by the minimized probable OOS reception at the receiver. The proposed adaptive technique uses stochastic model to perform two main functions. Firstly, it performs adaptive path ranking on the basis of probe results of E2E measurements that are performed as control signaling using ICMP packets. The E2E delay statistics are used to perform minimized OOS constrained scheduling of queued traffic on available interfaces; exhausting the best link first. Therefore, accuracy of inference process regarding link ranking constitutes the core challenge to achieve better multipath transport throughput. The path ranking is based on probe data of each flow.

\subsection{Adaptive Path Ranking (APR)}

In a multimode device equipped with multiple network interfaces; multiple E2E paths may be used to implement faster data transport services between the sender and receiver. The proposed APR performs core function of choosing best paths between source and destination during multi-path transmission of a flow. In order to ensure maximum in-order packet delivery, it is imperative to use links with matching delay statistics. The results achieved through probe signalling between source and destination provides the statistical data and delay distribution. The cumulative E2E delay distribution is a convoluted spectrum of delays of all paths. The right side tail of the distribution is, therefore curtailed to constraint E2E delay in an acceptable range. In order to further strengthen path ranking dependability; throughput and packet drop rate on path are also included in the path rank calculation. The unified impact of these three parameters increases reliability of the path rank indicator and improves throughput. We propose a simplified probing mechanism that sends a set of ICMP probe packets and recorded their E2E delay, round-trip time (RTT) and packet drop rate. These parameters are collected through ICMP messages and are then mapped over predefined decision tables generated through a Fussy Logic (FL) system. The table comprises of three bit binary pattern that produces rank in the range 0 to 7 (binary 000-111). Hence the link with a specific throughput will get its suitability index in the range of 0 to 7. Similarly; other tables map E2E delay and packet drop rating that produce suitability index in the ranges of 0 to 7. All these suitability indexes are than combined to get the link rank (also termed as weight) using Eqn. (1).

$$
W_{i}^{j+1, k}=\alpha T_{i}^{j, k}+\beta P_{i}^{j, k}+\gamma D_{i}^{j, k}
$$

Where $W_{i}^{j+1, k}$ is the rank (weight) of the path $i$ for scheduling packet $j+1$ of flow $k$ on link $i$. Similarly, $T_{i}^{j, k}, P_{i}^{j, k} \& D_{i}^{j, k}$ are throughput, packet drop rate and delays of link $i$ recorded for $j$ th packet of flow $k$; respectively. The $\alpha, \beta \& \gamma$ are scaling constant whose values range between 0 to 1 in a way that $\alpha+\beta+\gamma \leq 1$. 


\subsection{Multi-Server Scheduler (MSS)}

The weights derived through $A P R$ are used to generate schedules of traffic on multiple paths. Let we assume at any time $t$, there are $N_{p}^{t}$ number of bytes waiting for service in $p$ number of queues such that array $Q=\left\{q_{1}, q_{2}, \ldots, q_{p}\right\}$ provides lengths in bytes of each queue be given by $l_{i}^{t}$ i.e. $\left\{l_{1}, l_{2}, \ldots, l_{p}\right\}$. Each of these queues represents a session flow data. These queues are also prioritized according to their service requirements such that the priority of $q_{1}>$ $q_{2}>\ldots>q_{p}$. Further, there are $M$ paths available with aggregated capacity of the system as $C_{A}^{S}=\left\{c_{1}, c_{2}, \ldots, c_{m}\right\}$, with individual path capacities represented by $c_{i}^{t}$, drop rate as

$P=\left\{p_{1}, p_{2}, \ldots, p_{m}\right\}$ represented by $p_{i}^{t}$, and delay statistics of $D=\left\{d_{1}, d_{2}, \ldots, d_{m}\right\}$, represented by $d_{i}^{t}$. Assuming a work-conserving model, we use APR to provide weight of the link to find best link for transmitting data waiting for service in $Q$. Assuming $\tau$, a small time interval during which backlogged traffic status does not change while algorithm completes it scheduling. Table 1 shows pseudo-code listing of MSS algorithm that schedules traffic on best available links as per priority of queues.

The algorithm after evaluating $c_{i}^{t}, d_{i}^{t}, p_{i}^{t}$ calls $A L R$ function to find rank of each link in the first for loop of the algorithm. In the next step it finds out the best ranked link in $H_{k}^{W}$, where $k$ is the index of the link with best rank and $W$ is the rank of the link. The Next for loop controls complete scheduling of traffic queues according to their priorities, with highest priority queue served first, on best possible links. Before the termination of loop, the best ranked linked is selected again for processing remaining queues. It's important to mention here that $c_{i}^{t}, d_{i}^{t}, p_{i}^{t}$ are updated in separate functions working as background processes and APR is also activated outside the function for continuous update. The while loop (line 7-13) controls the transmission of waiting traffic over the best available paths. The APR is triggered only once in the life time of a session to avoid depletion of bandwidth for signaling.

It is noticeable that proposed scheduling is a non-round robin scheduling approach that maintains order of the path selection based on the status of path regarding its suitability with respect to delay, throughput and drop rate. The MSS does not iterate the link use unnecessarily as it keeps on transmitting on the same link till its capacity is depleted and rank remains the best. This approach proactively increases the chances of in-order delivery of packets at destination port In case a single link is good enough for a particular session the algorithm will avoid multipath transport.

Table 1. MSS algorithm in pseudo form.

\begin{tabular}{ll}
\hline 1 & Input:Q, $N_{p}^{t}, l_{i}^{t}, c_{i}^{t}, p_{i}^{t}, d_{i}^{t}$ \\
2 & Output: Traffic Distribution schedule for all paths \\
3 & For $i=1$ to $m ; ;$ No. of paths \\
4 & $W_{i}^{t}=A L R\left(c_{i}^{t}, d_{i}^{t}, p_{i}^{t}\right) ;$ Find the rank of path i \\
5 & Next $i ; ;$ Process next path \\
6 & $H_{k}^{W}=\max \left(W_{i}^{t}\right) ;$ Find path with best rank \\
7 & While $\left(H_{k}^{W}>0\right.$ and $\left.N_{p}^{t}>0\right) ; ;$ Proceed while path capacity and backlogged byte are non-zero \\
8 & for $i=1$ to $Q ; ;$ loop for all queues \\
9 & $S_{k}^{t}=S c h e d u l e l_{i}^{t}$ on Link $k ;$ Complete Queue scheduled \\
10 & Empty $l_{i}^{t} ; ;$ ith queue is empty now. \\
11 & $H_{k}^{W}=\max \left(W_{i}^{t}\right) ; ;$ Find new best capacity path \\
12 & End For $($ Next $i)$ \\
13 & End While \\
\hline
\end{tabular}




\section{Probability of Delay Violation}

_- Probability of delay violation( 16 flows) _—Probability of delay violation ( 32 flows)

- Probability of delay violation(48 flows)



$\begin{array}{lllllllllllllllllllllllll}1 & 3 & 5 & 7 & 9 & 11 & 13 & 15 & 17 & 19 & 21 & 23 & 25 & 27 & 29 & 31 & 33 & 35 & 37 & 39 & 41 & 43 & 45 & 47 & 49\end{array}$ \# of Packets

Figure 2. Plot of probabilistic E2E delay at varying traffic load.

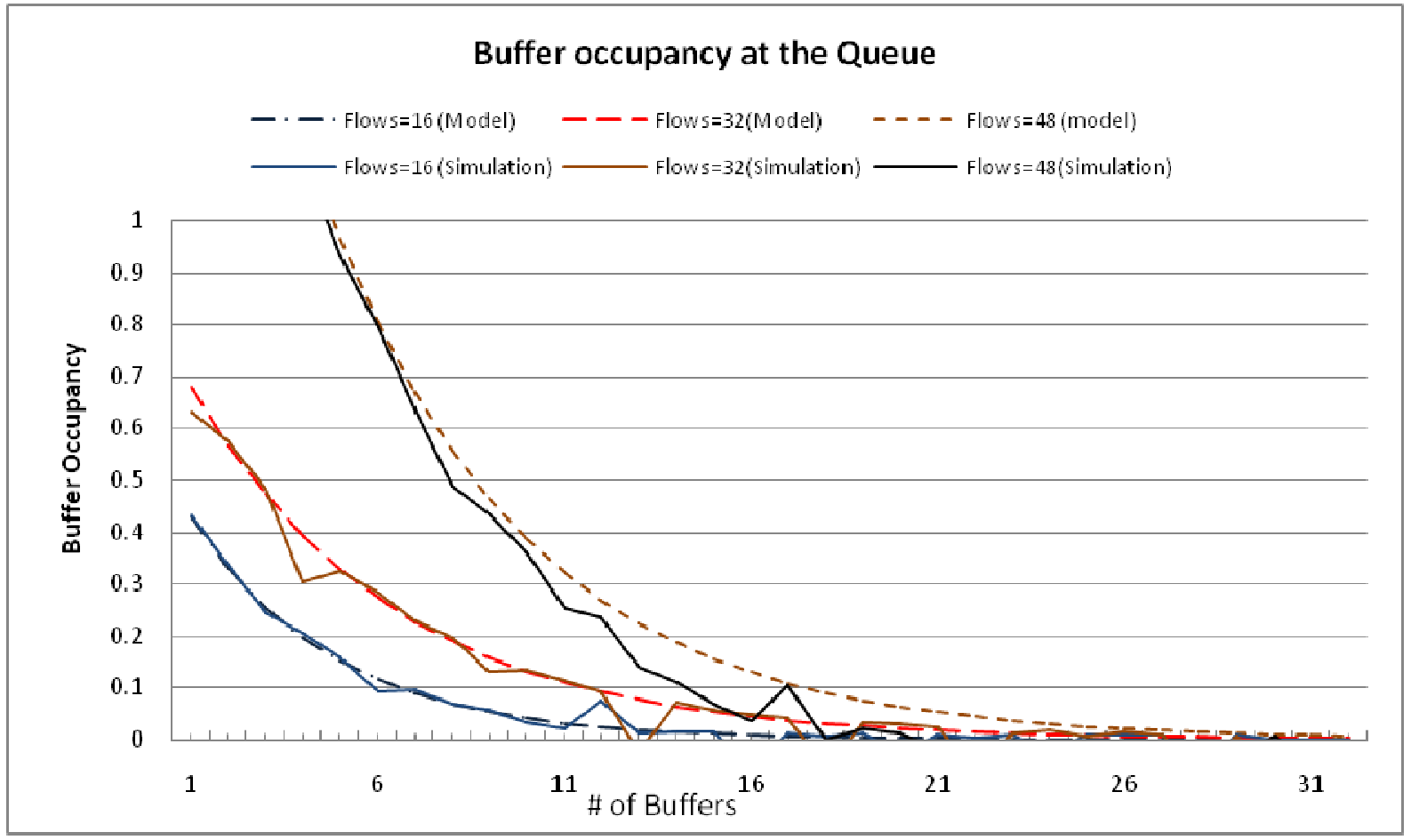

Figure 3. Buffer Occupancy behaviour at varying traffic load. 


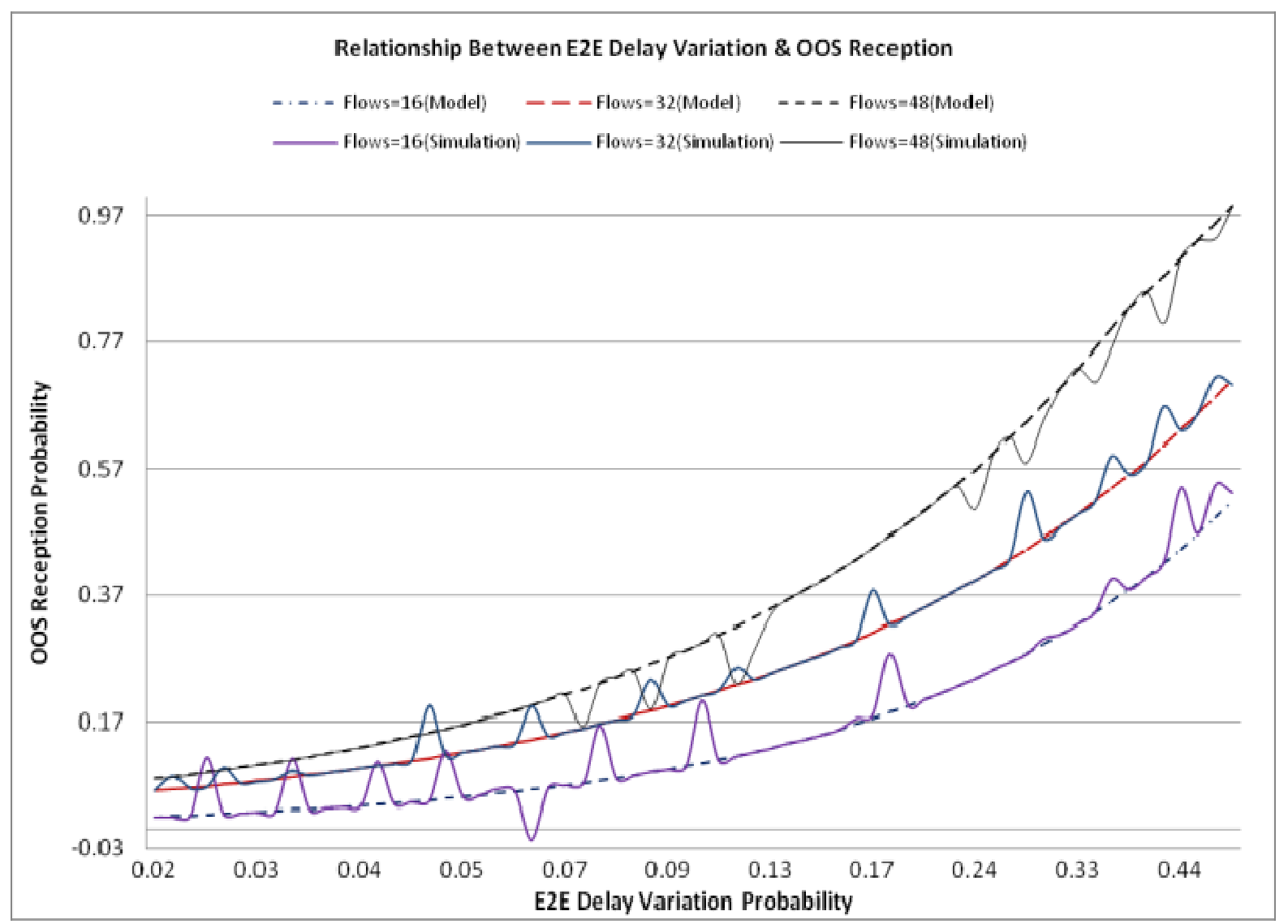

Figure 4. Relationship between probability of OOS reception and delay violation probability.

\section{Simulation Results}

Using the stochastic model presented in [2], the proposed multipath transport scheme is tested in ns-2 simulation environment [15]. The traffic load has been further increased to 48 sessions to test scalability of solution as compared to the results of our previous work presented in [2]. The simulation results are compared with the results of stochastic model. The simulation scenario is based on three paths between the multimode devices carrying three interfaces and matching sink devices. The three interfaces used are all WLANs; connected through different gateways. The intermediate nodes on each path are different in numbers as well. The intermediate nodes are also serving other transport sessions make even load on all intermediate nodes. Generally; the contribution of such traffic is referred to as cross load. The main metrics of interest for the experiment/simulation include probability of delay threshold violation, E2E delay distribution, probability of OOS arrival at sink and probability of buffer occupancy due to different rates of OOS arrival. The E2E delay reflects the suitability of proposed scheme for real-time applications; such as, voice and videos, whereas reduced re-order complexity reflects usefulness of proposed model and algorithm in reducing OOS reception of packet.

The probability of E2E delay violation is studied to analyse stability of path congestion state. Figure 2 shows this behaviour against increasing traffic load. It is noticeable that delay variation probability at the initial level of loads (up-to ten packets) increases sharply but stays in saturated region afterward. The stability of this region is a good indicator of stable congestion state of the path. The plot also indicates the upper bound of the delay variation probability that lies around $40 \%$. It highlights the fact that traffic load over a path may be kept to significantly moderate levels to minimize delay variations and increase the probability of in-order arrival of packets at the sink. The result also indicates that use of multipath transport can greatly reduce the queue length on each interface and subsequently delay variation probability may also be reduced.

Figure 3 plots buffer occupancy probability at varying buffer sizes. It is noticeable that the probability of buffer occupancy decreases with increase in number of available buffers. The experiment is repeated with 16, 32 and 48 traffic flows simultaneously leaving the sender. Ideally, we may assume infinite buffers to ensure sufficient memory space (not time) to hold OOS traffic to wait for their predecessor packets to arrive. In practice, we are constrained by limited buffer sizes that may be adjusted as per service requirements of the flows. The numerical and simulation results mostly coincide to support mathematical findings. The probability of buffer occupancy is calculated on the basis of OOS probability to keep tight binding between the two parameters. It also holds relationship with the E2E delay threshold violation to ensure consistency of the proposed model. The experiment has been performed at higher session load to test scalability of solution. 
Figure 4 shows a plot of OOS arrival at varying E2E delay probabilities. It may be noticed in the graph that probability of OOS arrival increases as both delay violation probability and traffic load increases. The OOS reception remains in low ranges of delay variations and at around 0.44 but shows divergent trends at higher loads. In figure 2, it was observed that the delay variation probability saturates at $40 \%$, hence the divergent trend is mainly attributed to the higher traffic loads. It is also noticeable that in parallel transport, there may be added time cushion for packets to wait at the receiver before being served to the applications without violating transport or application protocol thresholds. The numerical and simulation results show conformity with each other.

\section{Conclusions}

This paper presents a multi-path transmission scheme for tapping maximum benefit from multiple available network interfaces of a multi-homed device. It proposes a simplified scheduling model of multi-path transmission of traffic flows in resource constrained wireless network with respect to key performance metric of E2E delay. The major focus is on reducing impact of asymmetric path characteristics faced by a flow while traversing multiple paths. Adaptive link ranking based multi-server scheduling scheme is proposed to minimize the impact of path asymmetries. The proposed model is tested numerically as well as in event-triggered simulation environment. The results of the stochastic model are used to enhance performance of scheduling scheme that incorporates the critical parameters evolved through modeling process. The results indicate quite seamless integration of multiple links to achieve a single virtual channel comprising of combined capacity of all links. The results also signify importance of adaptive mode of monitoring E2E parameters to evolve any fruitful bandwidth aggregation scheme that maximizes aggregation gains. The critical nature of task requires fast convergence of ranking function to be effective in maximizing aggregation gains.

\section{References}

[1] Ahmad, Syed Zubair et al; "Adaptive Path Ranking Technique for Maximization of Gains of Bandwidth Aggregation over Heterogeneous Wireless Links." Proceedings of Seventh International Conference on Broadband, Wireless Computing, Communication and Applications. IEEE Computer Society, 2012.

[2] Zheng, Kun, et al. "An analysis of resequencing delay of reliable transmission protocols over multipath." Communications (ICC), 2010 IEEE International Conference on. IEEE, 2010.
[3] Ahmad, Syed Zubair, et al. "Analysis of Multi-Server Scheduling Paradigm for Service Guarantees during Network Mobility." Wireless Personal Communications 63.1 (2012): 177-197.

[4] Giacomazzi, Paolo. "Closed-form analysis of end-to-end network delay with Markov-modulated Poisson and fluid traffic, Published in." Computer Communications 32 (2009): 640-648.

[5] Janardhan R. Iyengar, Paul D. Amer, Randall Stewart. "Concurrent Multipath Transfer Using SCTP Multihoming Over Independent End-to-End Paths." IEEE/ACM Transaction on Networking 14, no. 5 (2006): 951-964.

[6] Serral-Gracia, René, Loránd Jakab, and Jordi Domingo-Pascual. "Out of order packets analysis on a real network environment." Next Generation Internet Design and Engineering, 2006. NGI'06. 2006 2nd Conference on. IEEE, 2006.

[7] Evensen, Kristian, et al. "A network-layer proxy for bandwidth aggregation and reduction of IP packet reordering." Local Computer Networks, 2009. LCN 2009. IEEE 34th Conference on. IEEE, 2009.

[8] Prabhavat, Sumet, et al. "Effective delay-controlled load distribution over multipath networks." Parallel and Distributed Systems, IEEE Transactions on22.10 (2011): 1730-1741.

[9] Kaspar, Dominik. "Multipath aggregation of heterogeneous access networks."ACM SIGMultimedia Records 4.1 (2012): 27-28.

[10] Lübke, Robert, et al. "NORA: An Integrated Network Measurement Tool for End-To-End Connections." 13th International Conference WWW/Internet. 2014.

[11] Jinghua, Y. A. N., et al. "Out-of-Sequence Traffic Classification Based on Improved Dynamic Time Warping." IEICE TRANSACTIONS on Information and Systems 96.11 (2013): 2354-2364.

[12] Prabhavat, Sumet, et al. "Load distribution using modified RED for multipath TCP communication." Information Technology and Electrical Engineering (ICITEE), 2013 International Conference on. IEEE, 2013.

[13] Wallace, T. Daniel, Khalim Amjad Meerja, and Abdallah Shami. "On-demand scheduling for concurrent multipath transfer using the stream control transmission protocol." Journal of Network and Computer Applications 47 (2015): 11-22.

[14] Liu, Qingyun, Wenzhong Feng, and Qiong Dai. "A Dynamic Strategy to Cache Out-of-Sequence Packet in DPI System." Trustworthy Computing and Services. Springer Berlin Heidelberg, 2013. 564-571.

[15] Wu, Jiyan, et al. "SPMLD: Sub-packet based multipath load distribution for real-time multimedia traffic. " Communications and Networks, Journal of 16.5 (2014): 548-558. 